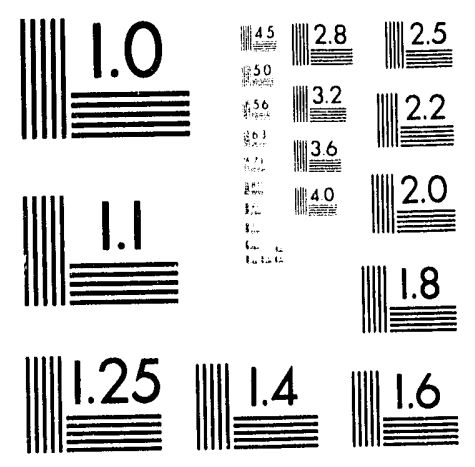



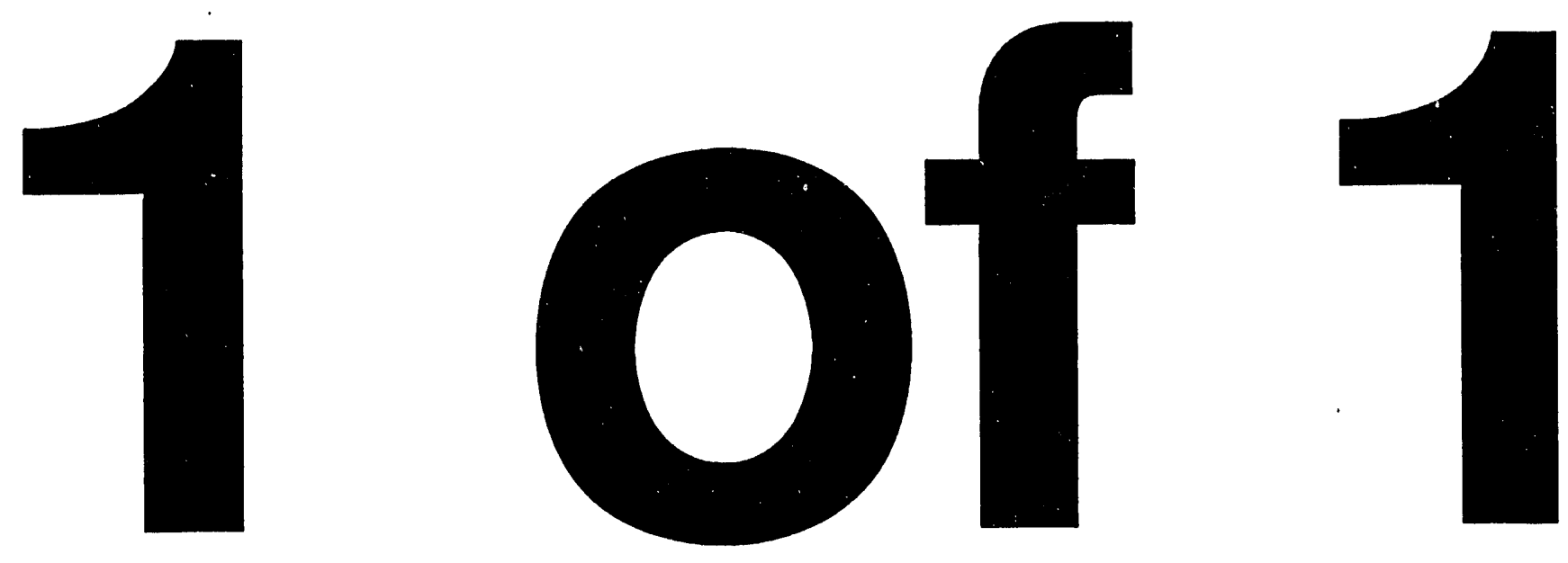
UCRL-CR-115002

B 160459

COT 281093

O S T

\section{A Prototype Distributed Audit System}

Debra L. Banning

Sparta, Inc.

August 1993

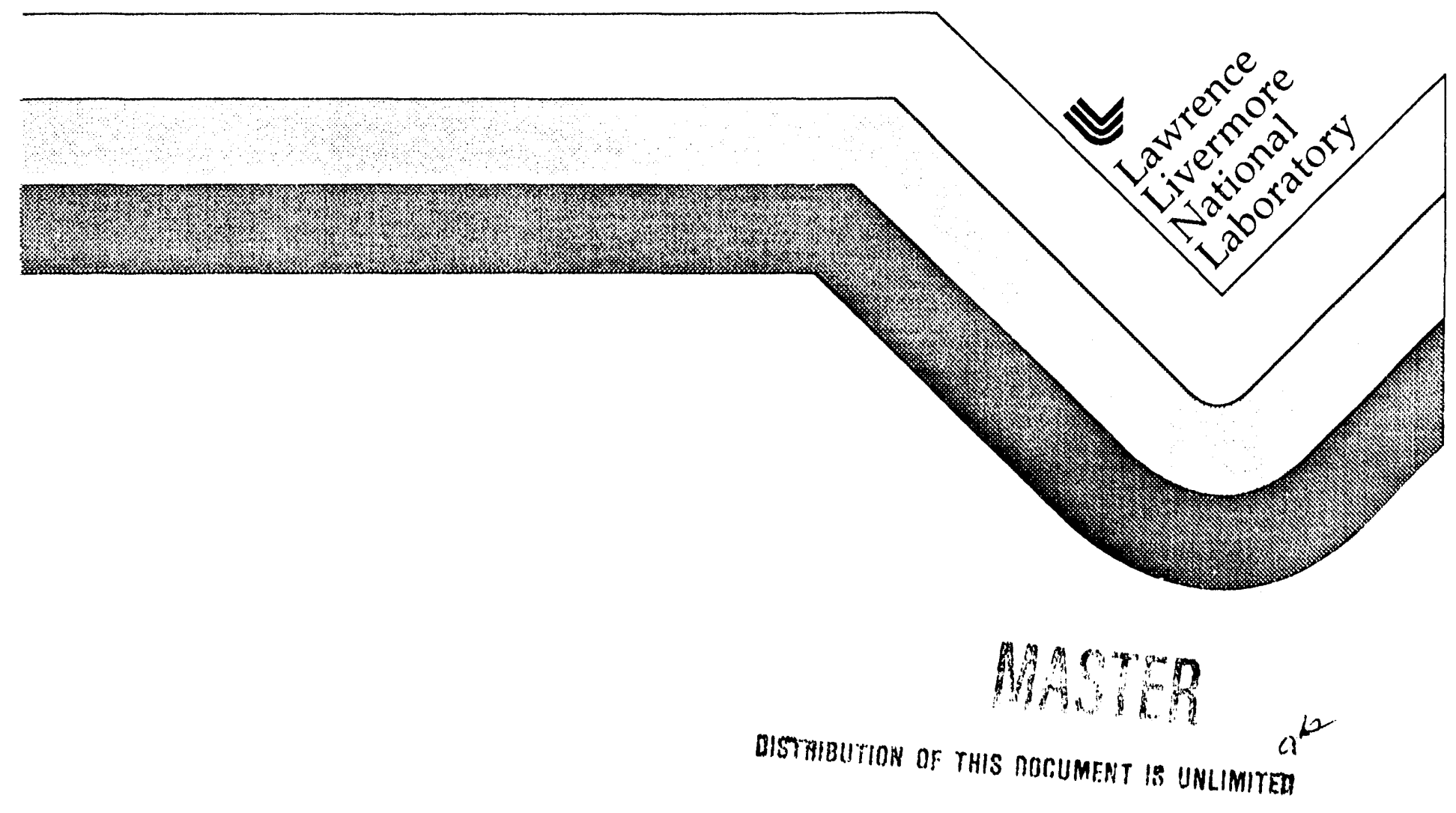




\section{DISCI.AINIER}

This document was prepared as un account of work spensored by an agencs of the Inited Stalius Government. Neither the United States Guternenent nor the University of California nor ans of their emplosece, makes any warranty. express or implied, or assumes any legal liability or responsibility for the accuracy, completeness, or usefulness of ans information. apparatus. product, or process disclosed, of represents that its use would not infringe privately ouned rights. Reference herein to ans specific commercial products, process, or service by trade name, trademark, manufacturer, or otheruise, deses not necessarily constilute or imply its endorsement, recommendation. or favoring b! the Lnited States Cowernment or the l'nisersity of California. The viens and opinious of authors expressed herein do not necessarily stale or reflect these of the Unifed States Government or the Iniversily of California, and shall not be used for adicrtising or product endorsement purposes. 
By

\author{
Debra L. Banning \\ SPARTA, Inc. \\ 615 Nash St. \\ El Segundo, CA 90245
}

\begin{abstract}
Security auditing systems are used to detect and assess unauthorized or abusive system usage. Historically, security audits were confinec' to a single computer system. Recent work examines ways of extending auditing to include heterogeneous groups of computers (distributed systems). This paper describes the design and prototype development of a Distributed Audit System (DAS) which was developed with funding received from Lawrence Livermore Laboratory and through the Master's thesis effort performed by the author at California State University, Long Beach. [1] The DAS is intended to provide collection, transfer, and control of audit data on distributed, heterogeneous hosts.
\end{abstract}

\title{
INTRODUCTION
}

The problem to be solved by the prototype is: "How can we control audit data amongst heterogeneous hosts in a network?" It has been a long time goal of intrusion detection designers to provide a means for determining abnormal activity amongst users on a stand alone system. However, when different types of systems become interconnected in a network, interoperability between the systems becomes lost. The primary need for interoperability lies in defining a standard commenications structure between different types of hosts. To do this it is also necessary to define a standard set of information that would be collected amongst different types of operating systems.

The DAS prototype is designed to provide this communications structure and framework for a standard definition of manageable audit data. The DAS prototype uses net,work management protocols and a graphical user interface to provide control over security audit data at distributed hosts from a centralized location. It is designed to take advantage of, but not duplicate, the many intrusion detection systems currently available or under development.

\section{AUDITING AS A NETWORK MANAGEMENT FUNCTION}

Network management protocols provide a mechanism for transmitting network performance information from remote nodes to a central collection point. The collection and reporting process for performance data and audit data are very similar. From review of the protocols it was determined that the network management protocols could be adapted for collecting, reporting, and transmitting audit information in a distributed network. This section gives a brief description of network management protocols and their applicability to distributed auditing.

\section{Introduction to Network Management}

Network management is accomplished by managers at local management stations and agents at remote managed nodes exchanging monitoring and control information via protocols and shared conceptual schema about a network and its components. The shared conceptual schema mentioned above is a priori knowledge about "managed objects" concerning which information is to be exchanged. Managed objects are abstractions of system and networking resources (c.g., a protocol entity, an IP routing table, or in this case, auditing resources) that are subject to management. Managed objects have attributes, operations, and notifications that are visible to managers. The internal functioning of the managed object is not visible to the manager. Currently, an agent is responsible for conversions between a managed system's intemal format of managed objects and the external format of managed objects (i.e., the form expected by the manager).

Work performed under the auspices of the U.S. Department of Energy by Lawrence l ivermore National Laboratory under Contract W-740.5-Eng-48. 
Using management services and protocols, a manager can direct an agent to perform an operation on a managed object for which it is responsible. Such operations might be to return certain values associated with a managed object (i.c., get a variable), to change certain values associated with a managed object (i.e., set a variable), or perform an action, such as self-test, on a managed object. In addition, the agent may also forward to the manager notifications generated asynchronously by managed objects (e.g., send updates periodically).

\section{Network Management Architecture}

The Network Management architecture described here consists of a Management Information Base (MIB) containing a list of managed objects, the International Organization for Standardization (ISO) Common Management Information Services (CMIS)/Common Management Information Protocol (CMIP) Manager and Agents [2]. The Managers and Agents exchange information based on the managed object definitions contained in the MIB, and the ISO network management protocols that facilitate the exchange of this information.

\section{CMIS/CMIP Manager and Agents}

The Common Management Information Services (CMIS) are the set of services provided by the Common Management Information Service Element. The Common Management Information Protocol (CMIP) supports these services. A CMISE-service-user is the part of an application process that makes use of the Common Management Information Service Element. An invoking CMISE-service-user, or "manager", may invoke a management operation. A performing CMISE-service-user, or "agent", is the process that performs a management operation invoked by a "manager."

C.MIS/CMIP supports a full set of basic services to facilitate standardized communication between CMIP managers and CMIP agents for monitoring and controlling network resources. The CMIP application can be run over a full OSI stack of protocols; however, it is also possible to run CMIP over a TCP/IP transport stack. In either case, CMIP always uses the ISO application layer services of the Association Control Service Element (ACSE) and the Remote Operations Service Element (ROSE). ACSE is used to establish and release associations between application entities. ROSE is the ISO equivalent of a remote procedure call. ROSE allows the invocation of an operation to be performed on a remote system. A CMIS/CMIP manager and agent applications could use adaptations of the ISO Common Management Information Service Element (CMISE) to exchange information and commands for the purpose of auditing.

CMISE provides to managers the ability to "multicast" operations to be performed on a group of managed objects. Through CMISE services, a manager can perform a single operation on a group of managed objects. A distributed audit mechanism could use such a service to assist in responding interactively to network attacks.

CMISE also provides facilities for a managed "agent" to send multiple linked responses to a manager. An Audit Agent (AA) could use this type of service to send detailed information to an Audit Manager.

\section{Management Information Base (MIB)}

A MIB is a list of managed objects, described in external format, which are considered useful for a particular application. A managed object is an abstract representation of a network resource that is subject to management. The objects of the MIB are defined in terms of their attributes that can be affected by management protocols. CMIP-based AAs instantiate the objects that are defined in the MIB and CMIP-based AMs operate on those MIB objects.

A MIB has been developed for the management of the Internet. The Internet MIB contains managed objects considered essential for either fault or configuration management. The managed objects can be read-only or readwrite, and help a manager determine the status of his network elements. Using the Internet MIB as a model, an Audit Management Information Base (Audit MIB) can be developed. 
$7 \quad$ The Audit MIB would define the managed objects upon which the CMIP-based Audit Managers may operate. The c definitions of these managed objects would be derived from end-user requirements. In the DAS, the end-user I requirements come from the needs of the audit analysis technique used at the AM. The Audit MIB must define I managed objects such that the AM's audit analysis tool would be capable of detecting abnormal behavior at the AA. ( Once the managed objects have been defined in the Audit MIB they are not removed. Commands issued from the 1 AM are used to create/delete instances of the defined managed objects such that information may be obtained about 1 them.

1 It is envisioned that an Audit MIB will encourage growth in distributed audit applications as has been seen in the area of Setwork Management. Implementations of AAs will have a standardized set of objects (the Audit MIB) that they will instantiate, therefore, it will not be necessary to create new agents for each new audit application. Likewise, implementors of distributed auditing applications will be able to concentrate on the specific application and not have to invent all of the supporting processes. Complete standardization of the Audit MIB is being addressed by standards committees and will take the cooperation of the computer security and vendor community.

Note that the Audit MIB object definition is written in Abstract Syntax Notation (ASN.1) as defined ioy ISO/CCITT [3]. This notation provides a mechanism for uniquely defining the semantics and syntax of the objects in a machine independent fashion that increases the scope of interoperability for the Audit MIB.

\section{DISTRIBUTED AUDIT SYSTEM DESIGN AND PROTOTYPE}

The DAS design consists of 4 primary components:

(1) Audit Agent (AA)

(2) Audit Manager (AM)

(3) Audit Data Communication System (ADCS)

(4) Audit Management Information Base (Audit MIB)

Figure 1 shows the overall DAS architecture. These components are described in the following sections.

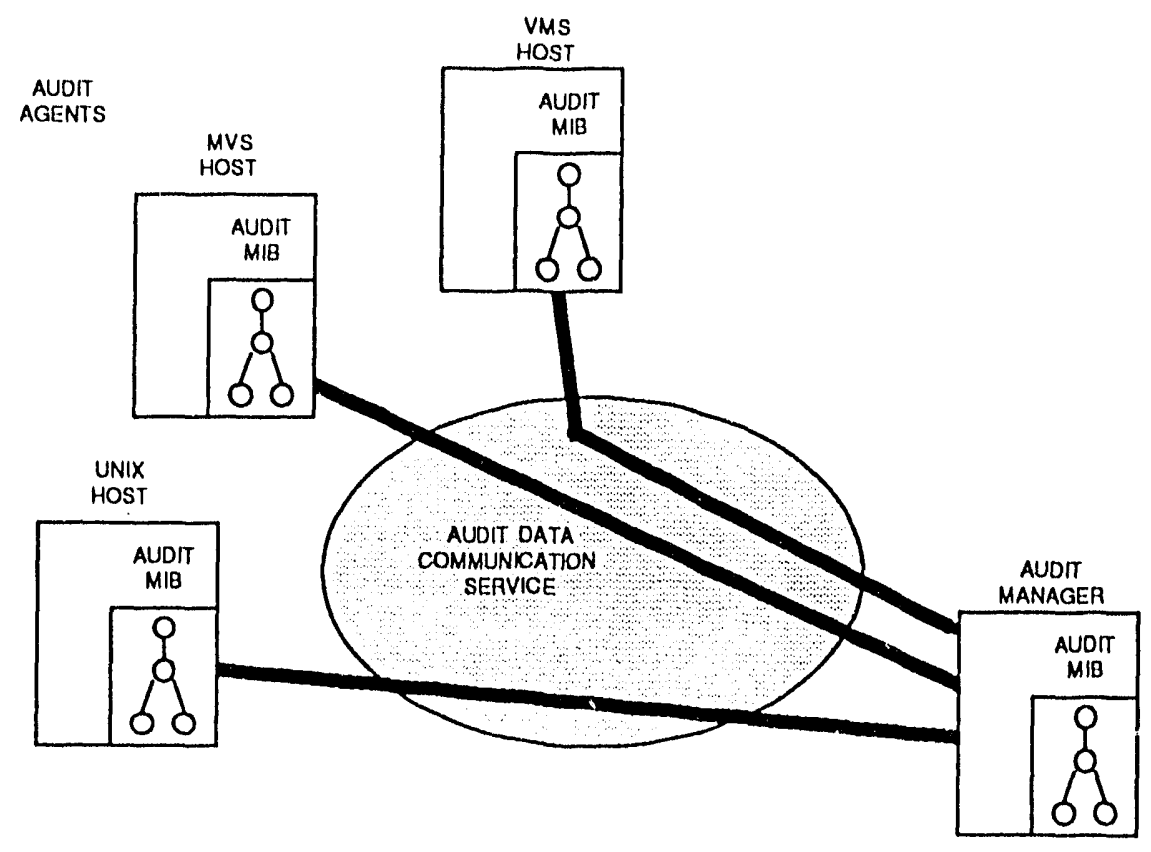

Figure 1. DAS Architecture 


\section{Audit Agent}

The Audit Agent (AA) application resides on all host systems being monitored in a network. The AA application provides the ability to retrieve collected audit data and transfer this audit data to the Audit Manager (AM).

At the AA it is assumed that audit data is collected from operating system and network interfaces and stored in some local form. The audit data storage is usually in the form of an audit trail but may also encompass administrative and special purpose local logs. The information maintained in the audit trail and local logs may actually consist of more information than is used by the managed objects. It is the responsibility of the AA application to retrieve the necessary attributes about a managed object from the stored data.

Most operating systems provide utilities to record accounting and some security-related information. The ultimate desire for the DAS would be to use systems that maintain C2 audit logs. However, this may be currently unrealistic though the Government has a goal of making all of their systems $\mathrm{C} 2$ compliant in the future.

For the prototype DAS the UNIX systems to be used do not have C2 audit logs. The only log maintained by the UNIX System V operating system is for accounting purposes. Security-related data will be obtained utilizing scveral UNIX utilities and from the accounting log.

For the prototype, the $/ \mathrm{var} / \mathrm{adm} / \mathrm{wtmp}$ file is used to obtain information concerning user login and logout. Changes in a user's effective ID is obtained from the / $\mathrm{var} / \mathrm{adm} / \mathrm{messages}$ which logs an entry every time a user does a "set user", "su", command. Information concerning the process a user is running is obtained from the $/ \mathrm{var} / \mathrm{adm} / \mathrm{paccl}$ filc.

To save overhead, the DAS prototype does not actually formulate a "security log." Instead user information is obtained dynamically from the available administrative logs, /var/adm/wtmp, /var/adm/messages, and $/ \mathrm{var} / \mathrm{adm} / \mathrm{pacct}$, and stored in temporary structures for response to user requests.

Once audit data is retrieved the AA is responsible for translating the local representation of the audit data (e.g., " $\mathrm{C}$ " structures, audit records) into an external form (i.e., managed object representation). As stated, earlier, managed objects are in ASN.1 definition that is understood by both the AAs and AMs.

In addition, the $\mathrm{AA}$ is capable of responding to $\mathrm{AM}$ commands to obtain additional audit information or modify a threshold that would trigger event reporting. The AA application is capable of responding to commands issued by the AM. The command set used is defined by the CMIS services provided under the ADCS.

Though in most cases the AM will request information about a managed object, it is desirable for information on some events (e.g., user login) to be sent asynchronously to the AM. This capability is called event reporting. In the prototype, AAs are capable of sending event reports on a defined set of events.

\section{Audil Manager}

The Audit Manager (AM) application resides on a centralized system that would act as a controller for the network. The AM is responsible for collecting and controlling audit data residing on all AAs under it's jurisdiction. The audit data is subjected to an analysis technique which could be performed by a security officer, an automated audit analysis tool or, most likely, a combination of the two. A large network may be divided into subnetworks with an AM for each subnetwork.

The AM is responsible for receiving audit managed object definitions from the AA and presenting them for analysis. The analysis process may be either an automated analysis technique (i.e., an Intrusion Detection System (IDS)) or a security officer. 
Audit data presented to the security officer will be via a Graphical User Interface (GUI). The audit data presented to the security officer may be raw data coming directly from the AA if an IDS is not used or it may be the results of the IDS after it has analyzed the AA's audit data.

The AM will be capable of controlling AA audit data. Control is necessary to increase granularity of auditing based upon results of analysis (e.g., obtain additional information about a suspicious user).

Issuance of control commands may be triggered either automatically by an audit analysis tool or by security officer interaction via the Security Officer Interface. The command structure to be used by the AM is provided by the $\mathrm{ADCS}$ as described in the next section.

\section{Audic Data Communication Service}

The Audit Data Communication Service (ADCS) provides the communication services necessary to transport messages between the Audit Agent (AA) and the Audit Manager (AM). The ADCS provides the ability to monitor and control network resources by transmitting command sequences between the AA and AM.

The communication component of the DAS will be provided by the International Standard (IS) Common Information Management Services and Protocol (CMIS/CMIP) [2]. Transfer of audit data and control commands will be accomplished using CMOT (Common Management Information Protocol Over TCP/IP) [4]. The objective of CMOT is to map the OSI management protocol architecture into the TCP/IP environment. CMOT follows the OSI model at the application layer, while using Internet protocols at the transport layer.

There are four primary commands provided by the CMIS services that the AM can use to control auditing at an AA. These commands are:

$\begin{array}{ll}\text { 1. } & \text { CREATE } \\ \text { 2. } & \text { DELETE } \\ \text { 3. } & \text { GET } \\ \text { 4. } & \text { SET }\end{array}$

The AM uses Create and Delete commands to affect instantiations of managed object classes on the AA. Both of these services can only be invoked in a confirmed mode in which case a reply is expected. The AM would issue a Create command to cause an instantiation of a managed object class which would allow information to be obtained about a managed object's attributes. The AM would issue a Delete command to cause an instantiation of a managed object class to be removed when no further information concerning the managed object is needed at the time. Since these commands do not affect the definition of the managed object in the Audit MIB, deleted instantiations of managed objects can be recreated when needed.

The AM would issue a Get command when additional information about a managed object's attributes is needed.

The AM would issue a Set command when it is necessary to associate a threshold with a managed object.

In addition to the above commands, the CMIS services provides the AM the ability to receive Event Reports from the AA which are used to describe an event about a managed object. These reports will be sent asynchronously (i.e., when some threshold has been reached).

\section{Audit Management Information Base}

The Management Information Base (Audit MIB) is a "conceptual repository of management information." It is an abstract view of all the objects in the network that can be managed. The Audit MIB is conceptual in that it does not carry any implications about the physical storage imain memory, files, databases, etc.) of management information. Therefore, the Audit MIB can consist of the administrative log, audit log, system files, and information gathered from the network and operating system interfaces. 
For the initial prototype, two Intrusion Detection Systems $[5,6]$ were reviewed to determine a common set of audited events that would be used to define a basic Audit MIB. Since both of the intrusion detection systems used were UNIX-based and the prototype DAS is UNIX-based, the classes for the Audit MIB are given in familiar UNIX terminology. The information defined in the Audit MIB can be obtained from other types of operating systems and the terminology will be modified to be more generic for future versions of the DAS.

The basic Audit MIB is shown in Figure 2. As can be seen in this figure, there are four classes defined under the audit class, i.e., the "Audit Control Class", the "Audit Process Table Class", the "Audit Record Table Class" and the "Suspicious Users Class".

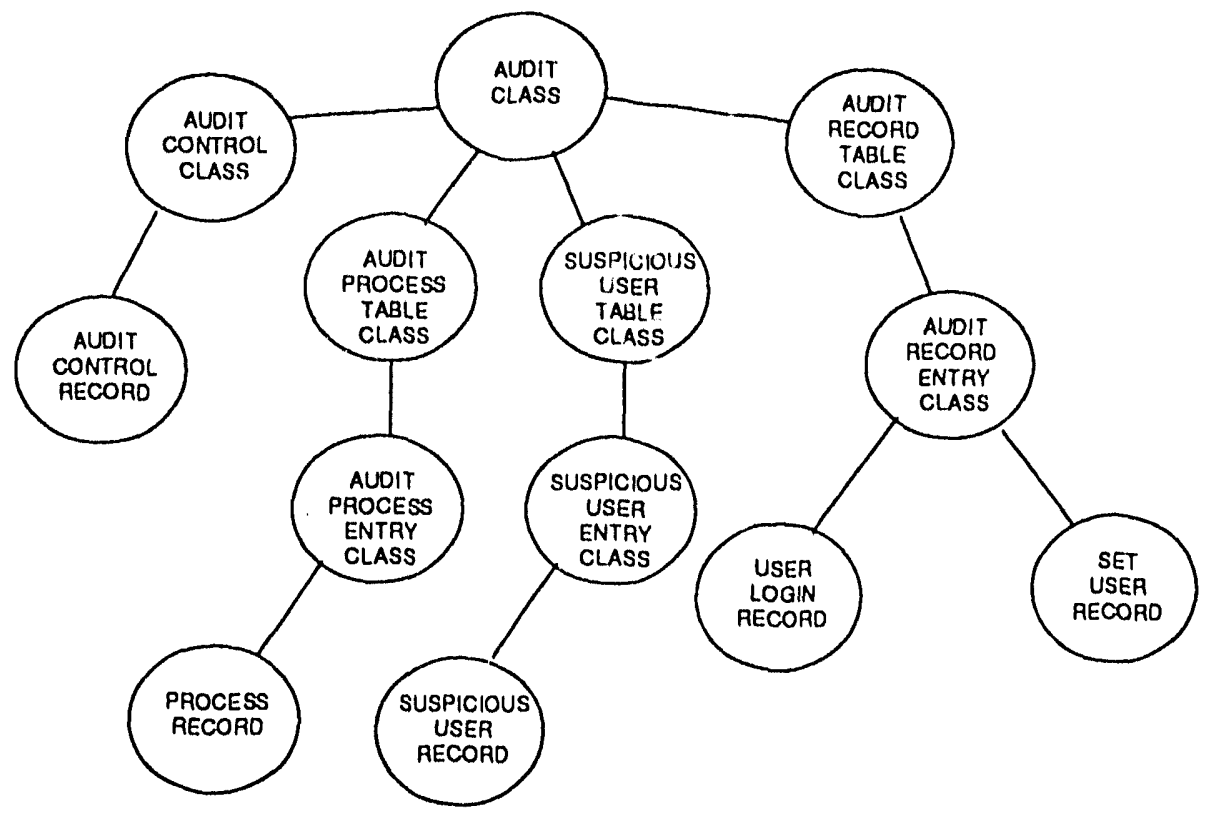

Figure 2. Audit MIB

The "Audit Control Class" manages the asynchronous Event Reporting capability. This class specifies what events or User IDs (UIDs) will trigger an event report. The current mode for the prototype is for Event Reports to be sent on all events and UIDs.

The "Audit Process Table Class" has the subclass "Audit Process Entry Class" defined which manages process information for individual users in the "process" record. The "Audit Process Table Class" manages a table of all "process" records. The "process" record defines attributes based on information obtained from the /var/adm/pacct file. This information is as follows:

User ID (UID)
Process start time
TTY name
Process ID (PID)
Process name

The "Audit Record Table Class" has the subclass "Audit Record Entry Class" defined which manages individual "user login" and "set user" records. The "Audit Record Table Class" manages a table of all "user login" and "set user" records. 
The "user login" and "set user" records definc attributes based on information obtained from the files /var/adm/wtmp and /var/adm/messages, respectively. This information is as follows:

User ID (UID)

Start/end time

TTY name

Host address

The record contains an additional "type" attribute which defines information specific to the particular record. For the "user login" record this attribute states whether the operation was a user "login" or "logout". For the "set user" record this attribute states whether the "set user" operation was "successful" or "unsuccessful".

The "Suspicious Users Class" is used to specify those users that should be monitored more closely. By defining this class the user is able to create records on suspicious users which by specifying "selectedUids" in the "Audit Control Class" would enable the user to receive Event Reports on only this group of users. On a large system where many Event Reports may come in every minute as users log on/off the system this granularity allows a user of the DAS to isolate the activity of a group of users.

\section{SECURING THE DISTRIBUTED AUDIT CAPABILITY}

In order for the distributed audit capability to be effective, it must be possible to ensure the security of the audit data being analyzed. Since audit data is intrinsically sensitive, it must be possible to ensure that it cannot be read as it crosses the network. In addition, since audit managing procedures must initialize audit recording and request retricval of sensitive data, it must be possible to verify that only validated procedures are making the requests. Also, the data being sent to an Audit Manager, either in response to a request or asynchronously, must be validated in order to develop reliable audit reports.

Several methods are being considered for providing the necessary security for the DAS. One method is to use the services to be provided by the Government Network Manage Profile (GNMP). The GNMP services are: authentication, access control, data confidentiality, data integrity, and non-repudiation of messages. Standards are currently being developed for these services with authentication and access control being given the highest priority.

A second method under consideration is to use the set of security protocols defined under an effort led by the National Security Agency (NSA) which could be used in conjunction with the ISO protocol suite. These security protocols would be conceptually "inserted" between various ISO protocol layers and would provide the security functions deemed to be required at certain layers in order to create a secure ISO stack. The SP4 security protocol would be inserted between the Network Layer (layer 3) and the Transport Layer (layer 4) [7]. The SP3 security protocol would be inserted between the Link Layer (layer 2) and the Network Layer (Layer 3) [8]. One of these protocols would be incorporated into the current CMIP stack. .

A third alternative being considered is to utilize CMIP as an application protocol and substitute an alternative transport protocol such as the Versatile Message Transport Protocol (VMTP) [9]. The ISO Development Environment (ISODE) currently supports CMIP over TCP/IP, which allows exploration of other transport services. VMTP uses a Public Key Encryption scheme and provides message authentication through a two step process.

Security was not included in the initial prototype of the DAS. These methods and cihers will be further researched and one method, or a combination of methods, will be incorporated in a future version of the DAS.

\section{PROTQTYPE QPERATION}

In 1992, a prototype DAS was developed which served as a proof-of-concept that the network management protocols could be used to provide a standard definition of audited events and to control audit data on remote hosts in a distributed environment from a central system. The DAS prototype provides a distributed auditing capability 
between two Audit Agents : ad one Audit Manager. Both the Agents and the Manlager are UNIX systems for the prototype.

From the Audit Manager the user is able to receive and control audit data at either Audit Agent via a user-friendly Graphical User Interface (GUI). The GUI uses XWINDOWS for a windows display. The prototype provides several windows for the user as shown in Figure 3. An "Agent Management" window will show which Audit Agent is currently being managed. A "Text Input" window allows the user to issue textual commands to the Audit Agent currently under management. Results to requests made by the DAS user are displayed in the "Text Input" window. One window for each agent is provided to display asynchronous Event Reports from the respective Audit Agent.

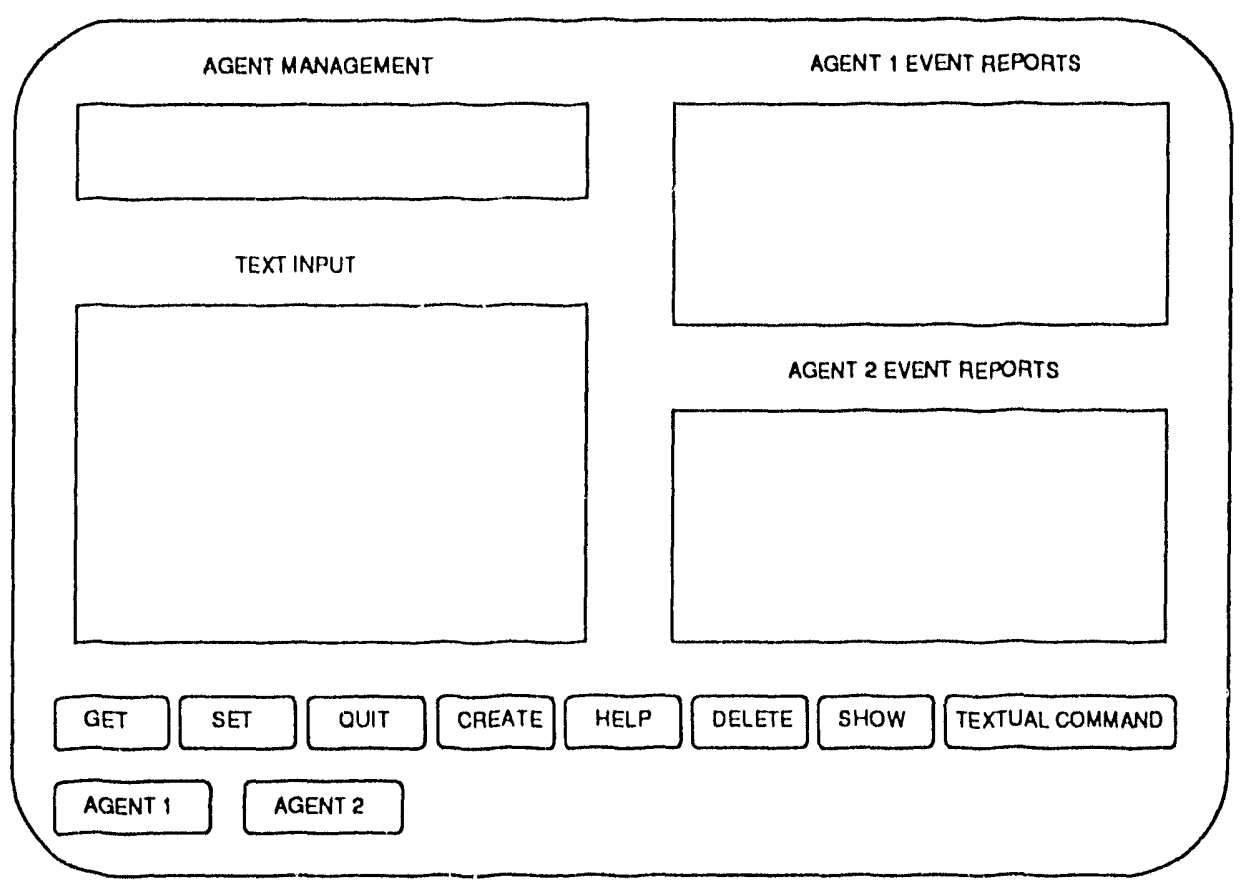

Figure 3. Graphical User Interface for the DAS Prototype

The commands provided in the prototype can be oivided into two categories: (1) control commands issued to the Audit Agent, and (2) commands that assist in the operation of the prototype. All commands are provided via mouse buttons. The control commands consist of:

\begin{tabular}{|c|c|c|}
\hline • & GET & obtain information about a managed object class \\
\hline & SET & change the value of an attribute \\
\hline & CREATE & creatc an instantiation of an object class \\
\hline & DELETE & delete an instantiation of an object class \\
\hline
\end{tabular}

The commands used to assist in the operation of the prototype include:

$\begin{array}{ll}\text { SHOW } & \text { display information about a managed object } \\ \text { HELP } & \text { display information about command syntax } \\ \text { QUIT } & \text { exit the prototype }\end{array}$

The security officer will use the above commands to obtain information about the managed objects defined in the baseline standard Audit MIB described carlier in this paper. 


\section{SUMMARY}

T To date there exists a few other projects that are attempting to address security auditing in a heterogeneous d) distributed network. DAS is unique in that it provides the solution by: (1) defining a standard definition of al audited events, and (2) using existing network management protocols for the transfer and control of audit data.

F Further enhancement of the initial DAS prototype is currently planned.

T The prototype DAS was incorporated into SPARTA's LAN. In most cases the audit data used by the DAS was o obtained either dynamically or from system files normally maintained on the systems. One exception was in the c) collecting of accounting information. An alternative method for collecting information derived from the a accounting utility is being explored since many UNIX systems do not use this utility due to the additional overhead it it causes. This change would not affect the overall DAS design, only the local implementation on UNIX systems. A Additional analysis of the effect on system performance will be performed during continued DAS development.

F Future modifications will also include the addition of different types of audit agents, including VMS and SUN OS C C2 audit agents. The addition of a SUN OS C2 audit agent would be in line with the needs of several well-known it intrusion detection systems.

I The initial prototype presents the audit data to the security officer for analysis. Enhancement of the prototype " would include integration with an existing intrusion detection system. The Distributed Intrusion Detection System ( (DIDS), being developed for the Air Force by the Lawrence Livermore National Laboratory, Haystack Laboratories, a and UC Davis, is a likely candidate for integration.

\section{REFERENCES}

1 1. Banning, Debra L., "A Distributed Audit System (DAS) Using Network Management Protocols," Masters Thesis, California State University--Long Beach, December 1992.

2 2. Information Technology--Open Systems Interconnection--Common Management Information Protocol-Part 1: Specification for CCITT Applications, November 24, 1990.

1 3. Specification of Abstract Syntax Notation 1 (ASN.1)--IS-8824, International Organization for Standardization, 1987.

4 4. Internet RFC 1155, The Common Management Information Services and Protocol over TCP/PP (CMOT), April 1989.

! 5. T. Lunt, A. Tamaru, F. Gilham, R. Jagannathan, C. Jallai, H. Javilz, P. Neumann, A. Valdes and T. Garvey, "A Real-Time Intrusion Detection Expert System (IDES), Final Technical Report, February 28, 1992.

$1 \quad 6 . \quad$ S. Smaha, "Distributed Intrusion Detection System (DIDS)--Preliminary Design," December 1990.

7. SDNS, Secure Data Network Systems, Security Protocol 4, SDNS Protocol and Signaling Working Group SP4 Sub-Group, SDN.401, 1988.

8. SDNS, Sccure Data Network Systems, Security Protocol 3, SDNS Protocol and Signaling Working Group SP3 Sub-Group, SDN.301, 1988.

9. D. Cheriton, VMTP: Versatile Message Transaction Protocol, Preliminary Version, January 1988. 


$$
[
$$

